\title{
Thermally Activated Energy Dissipation in Semi-crystalline Polymer Nanocomposites
}

\author{
Frank Gardea ${ }^{1}$, Bryan Glaz ${ }^{2}$, Jaret Riddick ${ }^{2}$, Dimitris C. Lagoudas ${ }^{1}$ and Mohammad Naraghi ${ }^{1 *}$ \\ ${ }^{1}$ Department of Aerospace Engineering, Texas A\&M University, 3409 TAMU, College Station, Texas \\ 77843-3409, USA \\ ${ }^{2}$ Vehicle Technology Directorate, US Army Research Laboratory, Aberdeen Proving Ground, Maryland \\ 21005-5066, USA
}

\begin{abstract}
In this manuscript, we demonstrate the concept of active damping in semi-crystalline thermoplastics which are reinforced with a percolated network of CNTs, where the damping of the composite was augmented considerably, controllably and reversibly via Joule heating. The Joule heating triggered relaxation mechanisms in the amorphous phase of the matrix. To this end, semi-crystalline poly ether ether ketone (PEEK) polymer and PEEK/carbon nanotube (CNT) composites were fabricated and their viscoelastic properties studied. The damping capability was experimentally tested by dynamic mechanical analysis. The polymer relaxation resulting from an increase in temperature, triggered by the Joule heating of the nanoparticles, demonstrated the potential for damping enhancement in the composite. A considerable enhancement in damping (by as much as 400\%) was achieved at a significantly lower relative loss in storage modulus (40\%), both caused by relaxation mechanisms in Joule heated samples. This enhancement in damping corresponds to a $150 \%$ improvement in the figure of merit for damping materials. The non-uniform temperature distribution in the sample was measured experimentally at the macroscale and estimated via continuum models at the microscale. It was concluded that non-uniform temperature distribution in the composite, especially at the microscale, had a large effect on the overall damping enhancement. Based on the microscale models, potential mechanisms by which the active damping can be enhanced are discussed.
\end{abstract}

Keywords: Active damping, viscoelastic damping, nanocomposites, PEEK

* M. Naraghi. Tel.: (979) 862-3323. Fax: 979-845-6051. E-mail address: naraghi@ aero.tamu.edu, Webpage: http://aero.tamu.edu/faculty/naraghi

\section{Introduction}

The ratio of loss to storage modulus, also known as $\tan \delta$, in polymers is strongly dependent on temperature, especially near the glass transition temperature, $T_{g}$. [1]. The remarkable temperature sensitivity of viscoelastic properties of a polymer can be utilized to dissipate undesired mechanical energy input to polymer-based composites in the form of vibration. This concept can in principle enable the development of advanced concepts such as active rotor vibration reduction leading to reduced operating

\section{$1 / 21$}


and maintenance cost [2-4]. This concept can also be used to enhance impact resistance, relieve stress concentration, improve crashworthiness and adjust dynamic performance of structures [5-7].

As a result, materials with inherent damping capabilities can replace the conventional vibration dissipation techniques (i.e. polymer-tapes [8]).

The highest energy dissipation in a polymer matrix often occurs near $T_{g}$, which corresponds to a state where polymer chains have gained sufficient thermal energy to initiate bond rotation. The significant rise in damping capability of polymers near $T_{g}$ originates from thermally activated molecular relaxations [1]. While $\tan \delta$ of polymers increases by a few orders of magnitude near $T_{g}$ compared to a glassy state, the increased damping capability is achieved at a loss in stiffness, evident in a sharp drop in storage modulus. The relative loss in stiffness near $T_{g}$ scales inversely with the concentration of the crystalline phase which remains solid during glass transition. Hence, energy dissipation can be significantly enhanced through relaxation of the amorphous phase near $T_{g}$, while the crystalline phase retains a considerable portion of the initial stiffness.

Similar to neat polymers, nanocomposites that are reinforced with nanomaterials, such as carbon nanotubes (CNT), demonstrate energy dissipation capability, partly arising from chain relaxation of the base polymer at increased temperatures. This mode of energy dissipation is in addition to energy dissipation due to matrix-CNT interfacial slippage [9], which is a direct consequence of unique matrixfiller interfacial characteristics in nanocomposites [9-11]. In addition, CNTs can form a percolated network within the matrix [12], which can be utilized to resistively heat the matrix (Joule heating) and thus to increase energy dissipation by thermally activating polymer chain relaxation. This thermo-electromechanical behavior, unique to composites with a percolated network of conductive fillers, allows for the active control and tuning of the dissipative viscoelastic mechanisms induced via external electric stimuli.

Previous studies have shown the potential of utilizing the thermomechanical response of polymers, activated via an external stimulus, to enhance damping. For example, Smith et al. developed a tuned-mass damper, in which damping was adjustable in a continuous range by controlling the temperature of the viscoelastic polymer through a resistive ribbon heater [13]. Polymer relaxation, accentuated by increasing the material temperature, has also been used to improve impact resistance and relieve stresses in carbon fiber reinforced composites, in which the fibers were used as resistive heaters [5, 6]. While the aforementioned studies have targeted energy dissipation in microfiber composites via resistive heating, a similar concept can also be applied to nanocomposites with potentially higher efficiency in terms of temperature rise time and uniformity within the material. That is mainly due to the higher surface-tovolume ratio of nanoscale fillers, which facilitate energy exchanges between filler and matrix.

In this manuscript, we investigated the active control of vibration damping in polymer-based nanocomposites which are reinforced with CNTs. The control of damping is by passing electric currents through the percolated network of CNTs, resulting in local heating of the polymer, and thus enhancing polymer chain relaxation. We focused our studies on poly ether ether ketone (PEEK) which is a semicrystalline polymer. Moreover, due to its remarkable thermal stability, mechanical and thermomechanical properties, PEEK has been introduced as a promising candidate for matrix phase in high performance composites for different applications including aerospace and biomedical industry such implants [14-18]. The semi-crystalline nature of PEEK is critical in our study as it limits the loss of stiffness during glass 
transition while $\tan \delta$ is thermally enhanced. Moreover, the enhancement in damping if achieved at an insignificant loss in complex modulus, can lead to an overall improvement of the figure of merit for damping materials (defined as the product of the absolute value of the complex modulus and tan $\delta$ ), thus, making the material a favorable choice for vibration damping [19]. However, it is to be noted that while a loss in stiffness may not be desired in most applications, in some cases, it can be used to desirably adjust the structural performance. For instance, it is shown that the reduction in stiffness, coupled with the increased energy dissipation, lowers seismic forces in civil structural components [7]. In addition, variable-stiffness members have been used to alter resonance frequency and suppress vibration [20]. We also developed analytical models to shed light on the origin of damping augmentation via electric signals.

\section{Experimental}

\subsection{Processing}

The matrix used in this study was poly ether ether ketone (PEEK) 450G, with a reported weight averaged molecular weight of 28000 [21], which was purchased in powder form from Victrex and kindly provided to us by Dr. Hung-Jue Sue at Texas A\&M University. The PEEK powder was first dehydrated in an oven at $150^{\circ} \mathrm{C}$ for $3 \mathrm{hrs}$. The neat PEEK samples were prepared by cast melting into an aluminum mold in a furnace at $400^{\circ} \mathrm{C}$ (above the melting point of $343^{\circ} \mathrm{C}$ ) for 5 minutes, followed by immediate quenching in a water bath. Quenching kept the crystallization of the PEEK polymer to a minimum. Subsequently, the samples were annealed to enhance crystallization. The time of annealing was varied, with a set of samples annealed at $240^{\circ} \mathrm{C}$ for 1,10 or 120 minutes.

PEEK/CNT composites were also fabricated to investigate the effect of CNTs on the thermomechanical properties of the composite and to study active damping. To fabricate the PEEK/CNT composites, the PEEK powder was first mixed with $1 \mathrm{wt} \%$ pristine single-walled CNTs (SWCNT) powder to form a homogeneous mixture. The HiPco ${ }^{\mathrm{TM}}$ SWCNT (Unidym, Inc.) consist of tubes with a diameter of 0.8-1.2 nm and a length of $100 \mathrm{~nm}-1 \mu \mathrm{m}$. The powder mixture of SWCNT/PEEK was then melt-casted at $400^{\circ} \mathrm{C}$ for 5 minutes followed by quenching in a water bath. Nanocomposite samples were then annealed at $240{ }^{\circ} \mathrm{C}$ for 1 minute. Samples for active damping experiments were prepared by attaching copper wire electrodes to the sample ends using high purity silver paint, minimizing joule heating at the electrode-sample contact point.

\subsection{Material Characterization}

Differential scanning calorimetry (DSC) was conducted on all samples to obtain the phase transition temperatures and the degree of crystallinity. The temperature sweep range was $25^{\circ} \mathrm{C}$ to $375^{\circ} \mathrm{C}$ (from below the glass transition temperature to above the melting point) at a heating rate of $10^{\circ} \mathrm{C} / \mathrm{min}$. The degree of crystallinity was measured by comparing the enthalpy of fusion of the totally crystalline polymer to the difference between the enthalpy of fusion at the melting temperature and the enthalpy of crystallization [22]. The latter was taken as $130 \mathrm{~J} / \mathrm{g}$ [23].

The energy dissipation capability of neat PEEK and PEEK/CNT composites was measured using a TA Instruments RSAIII Dynamic Mechanical Analyzer (DMA). The damping was quantified as the ratio of the loss to storage modulus ( $\tan \delta$ ). The temperature sweep range during the DMA experiments was $25^{\circ} \mathrm{C}$ to $200^{\circ} \mathrm{C}$ at a rate of $10{ }^{\circ} \mathrm{C} / \mathrm{min}$, which was applied at a dynamic strain amplitude of $0.01 \%$ and a 
frequency of $1 \mathrm{~Hz}$. The strain offset was set to zero and the magnitude of the dynamic strain was selected to avoid sample buckling during the compressive half-cycle.

To perform active damping experiments on PEEK/CNT composites, various voltages were applied to the sample during the DMA experiments, Figure 1. As shown in the figure, the setup consists of the sample gripped to the DMA tensile grips with the wire electrodes extending out of the sample. Each sample was $0.6 \mathrm{~mm}$ thick, $2.5 \mathrm{~mm}$ wide and more than $40 \mathrm{~mm}$ long. The distance between electrodes was $38 \mathrm{~mm}$, and the gage length (distance between mechanical grips) was $15 \mathrm{~mm}$. Electric power was applied to the sample via an Agilent 33220A waveform generator and a TREK 609E-6 high voltage amplifier. A sinusoidal wave signal of $1 \mathrm{kHz}$ at a specific voltage was applied to the sample. The applied voltage, Vrms, in the range of 0 to $101 \mathrm{~V}$, corresponded to an input power varying from $0-0.017 \mathrm{~W} / \mathrm{mm}^{3}$. A Testo 885 Thermal Imager was used to continuously monitor the temperature of the sample during dynamic testing. Dynamic testing was performed upon reaching a steady state temperature in the sample at an applied dynamic strain amplitude of $0.01 \%$ and a frequency of $1 \mathrm{~Hz}$. The frequency of the applied voltage $(1 \mathrm{kHz})$ was significantly higher than that of the DMA test frequency in order to assure negligible variations in temperature during each cycle of mechanical loading. Potential thermal degradation of the sample was also studied by comparing the viscoelastic properties of the sample before and after Joule heating, both at zero input power.

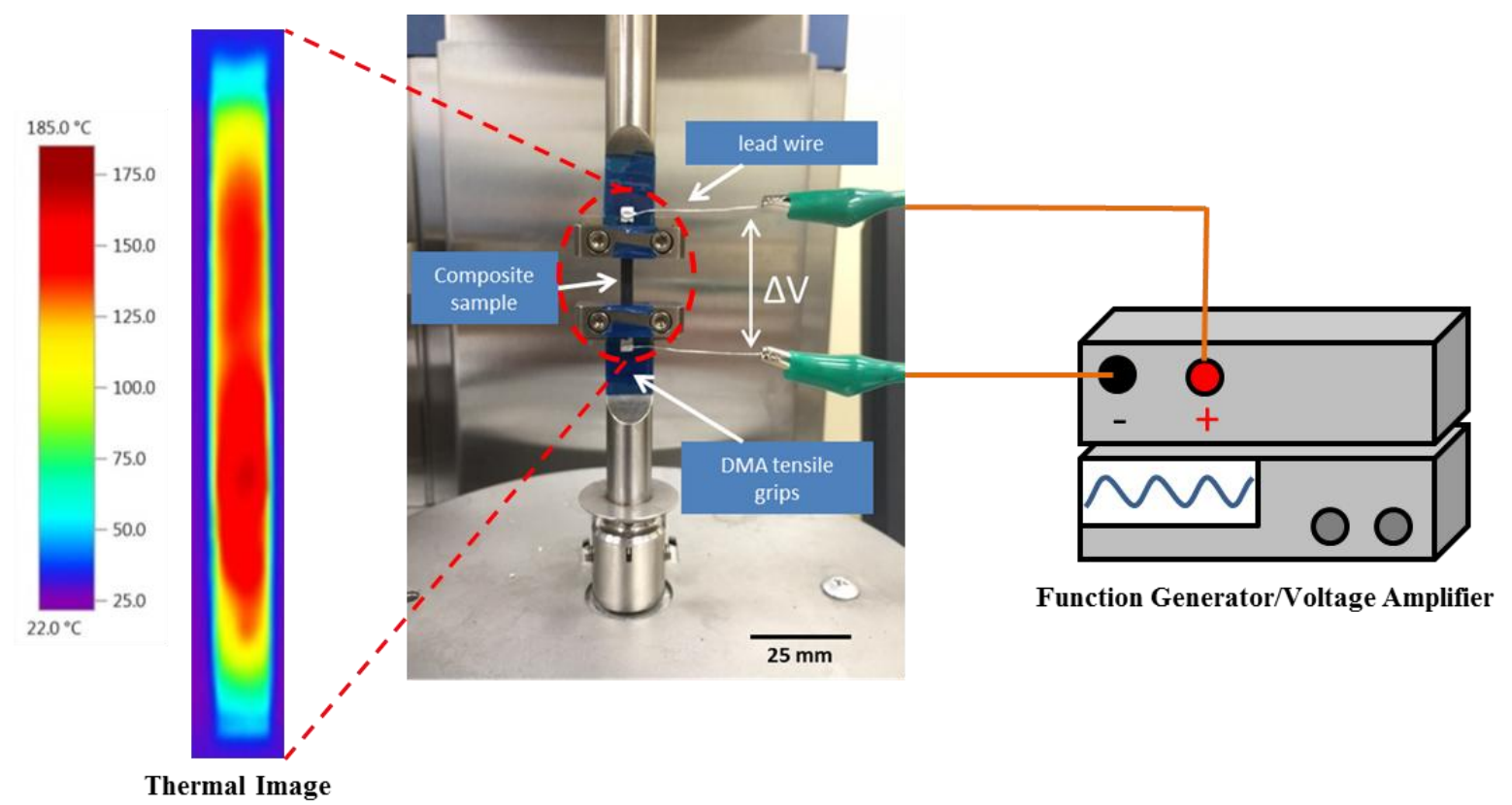

Figure 1. Test set-up showing a composite sample gripped to the DMA tensile testing machine. Copper wires were used as electrodes for applying the potential difference in the sample using a function generator and a voltage amplifier. The sample temperature was captured with a spatial resolution of $\sim 0.5 \mathrm{~mm}$ using a Thermal Imager. 


\section{Continuum Modeling of Active Damping in Nanocomposites}

The concept of active damping demonstrated in this study, can be described as accelerating the stress relaxation mechanisms in polymers via Joule heating. However, a critical requirement for effective implementation of this concept is the achievement of uniform temperature rise across the polymer phase in the sample. This is because the peak of $\tan \delta$ around $T_{g}$ in most polymers is a sharp peak with a full width at half maximum (FWHM) of only about $\sim 5-20^{\circ} \mathrm{C}$.

The temperature non-uniformity in Joule heated samples can arise from two different mechanisms which occur at two different length scales. In the macroscale, the sample heat loss to the environment via convection in air and conduction to the grips will induce a temperature gradient near the edges. Moreover since the heat in the sample is generated within CNTs and transferred from them to the surrounding matrix, local temperature gradients in the microscale around the CNTs are also expected. We investigated the effect of these temperature gradients on damping via continuum models by using the approaches outlined in this section. To take into account the effect of temperature gradient in the macroscale on damping, analytical solutions and finite element analysis (FEA) were used with experimental temperature measurements during Joule heating as input. Our analysis of the local microscale temperature gradients were based on analytical solutions of heat transfer in steady state from a heat source (Joule heated CNTs).

\subsection{Effect of Macroscale Temperature Non-uniformity on Damping: Analytical Models}

To study the effect of non-uniform temperature distribution in the composite at the macroscale, the temperature dependent viscoelastic properties obtained experimentally were used as input into a theoretical model using both Voigt and Reuss model structures [24]. To this end, the composite is assumed to be composed of several segments each with a narrow temperature distribution (approximated as a single-valued temperature), and thus a single-valued set of storage and loss moduli, Figure 2 . The assigned moduli are taken from DMA experiments in which the temperature of the sample is raised uniformly in a DMA oven (similar to a "heat bath" experiment). Given the symmetry of the boundary conditions with respect to the geometrical symmetry lines of the sample, the segments can be considered to be connected mechanically to each other in series/parallel or a combination of these. The Voigt and Reuss structure models can then be used to calculate the effective tan $\delta$ of the sample by taking into account the temperature non-uniformity. The effective viscoelastic properties of Voigt and Reuss models are briefly discussed here. 
(a)

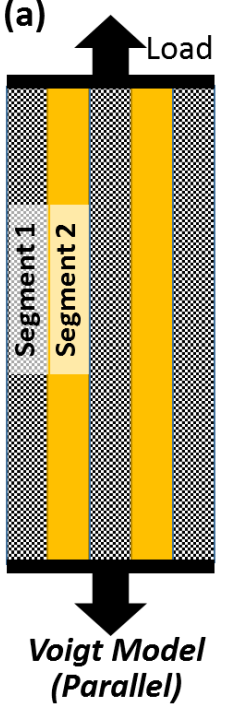

(b)

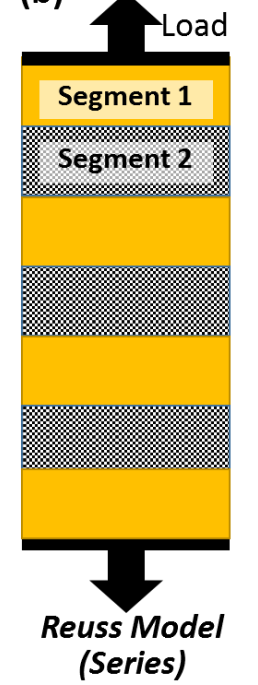

(c)

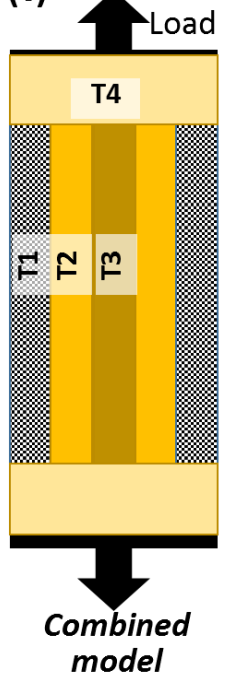

(d)

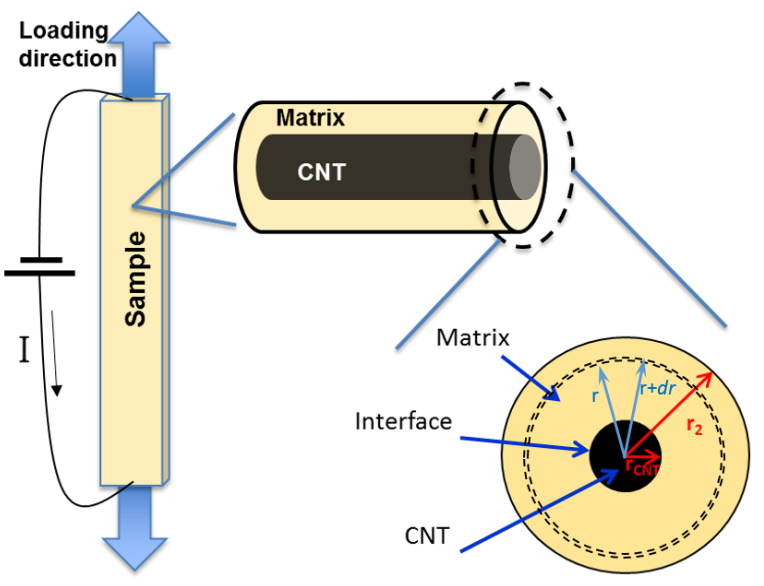

Figure 2. (a) Voigt parallel structure resulting in isostrain deformation, and (b) Reuss series structure resulting in isostress deformation. (c) An example of a sample assumed to be composed of segments each with a different temperature, and thus different material properties. The temperature is expected to be different near the edges and grips due to heat loss to grips and air. (d) Schematic showing the building block of a composite consisting of a CNT, interface, and matrix for the purpose of estimating the local heat transfer in the nanocomposite.

In a layered two-phase composite with a Voigt structural arrangement, Figure 2a, each phase experiences the same strain. Thus, the composite modulus follows the rule of mixtures. Using the correspondence principle [25], the elastic relation is converted into a viscoelastic relation resulting in Eq. (1), where $E_{c}^{*}$ is the composite complex modulus, $E_{1}^{*}$ is the complex modulus of the first phase, $E_{2}^{*}$ is the complex modulus of the second phase, and $v_{1}$ and $v_{2}$ are the volume fractions of phase 1 and phase 2 , respectively. The complex modulus is defined as $E^{*}=E^{\prime}+i E^{\prime \prime}$, where $E^{\prime}$ is the storage modulus, $E^{\prime \prime}$ is the loss modulus, and $\tan \delta=\frac{E^{\prime \prime}}{E^{\prime}}$.

$$
E_{c}^{*}=E_{1}^{*} v_{1}+E_{2}^{*} v_{2}
$$

Thus, from the ratio of the real and imaginary parts, $\tan \delta_{c}$, the loss tangent of the composite, is given by Eq. (2):

$$
\tan \delta_{c}=\frac{v_{1} \tan \delta_{1}+v_{2}\left(\frac{E_{2}^{\prime}}{E_{1}^{\prime}}\right) \tan \delta_{2}}{v_{1}+\left(\frac{E_{2}^{\prime}}{E_{1}^{\prime}}\right) v_{2}}
$$

As for the Reuss structure, Figure $2 b$, the layered phases are placed in series and thus experience the same stress (isostress). This structure follows the inverse rule of mixtures shown in Eq. (3). The $\tan \delta_{c}$ can be expressed as Eq. (4).

$$
\frac{1}{E_{c}^{*}}=\frac{v_{1}}{E_{1}^{*}}+\frac{v_{2}}{E_{2}^{*}}
$$

Thus, from the ratio of the real and imaginary parts $\tan \delta_{c}$ is given by Eq. (4): 


$$
\begin{aligned}
& \tan \delta_{c} \\
& =\frac{\left(\tan \delta_{1}+\tan \delta_{2}\right)\left(v_{1}+v_{2} \frac{E_{1}^{\prime}}{E_{2}^{\prime}}\right)-\left(1-\tan \delta_{1} \tan \delta_{2}\right)\left(v_{1} \tan \delta_{2}+v_{2} \tan \delta_{1} \frac{E_{1}^{\prime}}{E_{2}^{\prime}}\right)}{\left(1-\tan \delta_{1} \tan \delta_{2}\right)\left(v_{1}+v_{2} \frac{E_{1}^{\prime}}{E_{2}^{\prime}}\right)+\left(\tan \delta_{1}+\tan \delta_{2}\right)\left(v_{1} \tan \delta_{2}+v_{2} \tan \delta_{1} \frac{E_{1}^{\prime}}{E_{2}^{\prime}}\right)}
\end{aligned}
$$

\subsection{Effect of Macroscale Temperature Non-uniformity on Damping: Finite Element Analysis}

To assess the accuracy of the above presented analytical solutions, finite element analysis (FEA) was also performed, which takes into account global non-uniform temperature distribution in the composite. The analysis involves the use of the commercial finite element software package, ABAQUS. A 2-D plane stress model was implemented with biquadratic plane stress quadrilateral elements (CPS8). The full-field temperature distribution in the sample, taken from image processing of the experimental thermal images, was input into the model as a predefined temperature field. The temperature of each point was maintained throughout the simulation (Steady state). To each element a storage and loss modulus was assigned which was a function of the temperature of the element, as identified from the DMA experiments. It was assumed that the Poisson's ratio was not a function of temperature in the temperature ranges studied.

\subsection{Effect of Microscale Temperature Non-uniformity on Damping}

The local heat transfer in a CNT composite was modeled in what we considered the building block of the material: a single CNT axisymmetrically surrounded by matrix. The CNTs were considered to be straight rods with uniform diameters and identical dimensions, homogeneously dispersed in the matrix. The cross-section of the building block is shown in Figure 2d. Moreover, the CNTs were treated as volumetric heat sources which transfer heat to the matrix through the CNT surfaces. Given the high aspect ratio of the CNTs (100-1000), it was assumed that the heat transfer was mainly in the radial direction.

For a given heat flux through the interface of the nanotube and the matrix, there is a finite temperature discontinuity along the interface. The heat generated per unit volume of the nanocomposite, $q_{v}$, is $J^{2} \rho$ where $J$ and $\rho$ are the average current density and resistivity in the composite. The total heat generated in the CNT is $q_{v}$ times the volume of the building block. This input heat can be modeled as an input flux, $q_{s}$, from the outmost shell of CNTs to the matrix (heat generation power per unit area) as:

$$
q_{s}=\frac{q_{v} r_{C N T}}{2 v_{f}}
$$

where $r_{C N T}$ is the radius of the CNT and $v_{f}$ is the CNT volume fraction. The input flux, $q_{s}$, in Equation (5) is calculated by multiplying the average volumetric heat of the building block by the volume of the building block divided by the surface area of the CNT for a unit length of the building block $\left(2 \pi r_{C N T}\right)$. The volume of the building block is calculated as the volume of the CNT (per unit length) divided by the volume fraction. It is assumed that there is a continuous heat flux in the radial direction, at the CNT-

matrix interface. Due to conservation of energy, at steady state the total heat through each radial cross section around the CNT will be the same, hence, the heat flux at a radius $r$ around the CNT will be: 


$$
q(r)=\frac{q_{s} r_{C N T}}{r}
$$

Therefore, the temperature drop across the interface will be

$$
\left(T_{C N T}-T_{I}\right)=\frac{q_{s}}{h_{\text {int }}}
$$

where $h_{\text {int }}$ is the interfacial thermal conductance, $T_{C N T}$ is the temperature of the CNT, and $T_{I}$ is the temperature of the polymer at the boundary of the CNT and matrix. Moreover, considering a thin rim of polymer with thickness of $d r$, located at a radius $r$, the temperature will change by $d T$ by moving along the rim in the radial direction (Figure 2(d)) such that:

$$
d T=\frac{q(r)}{k_{m}} d r
$$

where $k_{m}$ is the thermal conductivity of the matrix. As a result, the temperature of the CNT can be expressed in terms of the temperature of the matrix, $T_{0}$, at the boundary, $r_{2}$, by integrating Eq. (8) and adding the temperature change across the interface from Eq. (7), as calculated in Eq.(9):

$$
T_{C N T}-T_{0}=\frac{q_{s}}{h_{\text {int }}}+\frac{q_{s} r_{C N T}}{k_{m}} \ln \left(\frac{r_{2}}{r_{C N T}}\right)
$$

Eq. (9), shows the contribution of the interface (first term in the right-hand side of the equation) and the contribution of the matrix (second term) to the overall temperature change between the CNT and the outside boundary. Even if the temperature distribution is uniform in the macroscale, according to Eq. (9), in the smaller length scales which corresponds to average spacing between CNTs in a nanocomposite (tens of nanometers to several tens of microns), the temperature may not be uniform, adversely influencing active damping control.

\section{Results and Discussion}

\subsection{Crystallization of Poly Ether Ether Ketone}

The effect of annealing time at $240^{\circ} \mathrm{C}$ on thermal properties of neat PEEK samples was studied via scanning differential calorimetry (DSC), Figure 3a. Prior to annealing, there is a clear shift in heat capacity, indicating a glass transition at $145.8^{\circ} \mathrm{C}$. After annealing for 1 minute, the $T_{g}$ shifts considerably by about $14^{\circ} \mathrm{C}$ to $159.3^{\circ} \mathrm{C}$. As annealing time is further increased from 1 minute to $120 \mathrm{~min}, T_{g}$ further increases but rather insignificantly from $159.3^{\circ} \mathrm{C}$ to $160.5^{\circ} \mathrm{C}$. In addition, prior to annealing, samples show an exothermic peak at $174^{\circ} \mathrm{C}$, corresponding to the crystallization during the differential scanning calorimetry experiment. After annealing only for 1 minute (the lowest duration of annealing in this study), this peak exothermic disappeared, indicating rapid crystallization of PEEK during such short periods of annealing.

The first endothermic peak (as the temperature is increased in DSC) is observed at $\sim 252^{\circ} \mathrm{C}$ in samples which were annealed for 10 minutes or more. This peak shifts to higher temperatures and increases in magnitude as the annealing time is increased. The second endothermic peak, which is considerably stronger than the first and was observed in all the samples regardless of the duration of annealing, occurs at a temperature of $\sim 343^{\circ} \mathrm{C}$. These two peaks correspond to the melting of two types of crystalline structures within PEEK and are referred to herein as minor and major endothermic peaks, respectively. 
The degree of crystallinity corresponding to each crystalline phase calculated from DSC curves is summarized in Table 1. As shown in the table, the degree of crystallinity of the minor crystalline phase increases from $1.56 \%$ to $2.48 \%$ for an annealing period of 10 and $120 \mathrm{~min}$, respectively. In comparison, the degree of crystallization (which is mainly the major crystalline phase) increased from $14.62 \%$ for the sample with no annealing to $31.88 \%$ for the samples which were annealed at $240^{\circ} \mathrm{C}$. Hence, the minor crystalline phase has a considerably slower crystallization dynamics compared to the major one. Moreover, the increase in $T_{g}$ from $145.8^{\circ} \mathrm{C}$ to $159.3^{\circ} \mathrm{C}$ as a result of annealing correlates strongly with the degree of crystallization of the sample. Thus, it is attributed to the loss of mobility of polymer chains as they are partly anchored inside the crystalline domains.

It is interesting to note that the formation of the minor crystalline phase is observed only in samples in which the major peak (at $\sim 343^{\circ} \mathrm{C}$ ) is grown to nearly a saturation (consider the relative changes in the degree of crystallinity of two phases as a function of annealing time in Table 1). In other words, the formation of the major crystalline phase precedes the minor phase. Hence, it is suggested that the minor crystalline phase forms in the regions of amorphous material which are trapped within the lamellae, thus leading to two distinct crystal populations [23, 26, 27]. In order to avoid the complications related to interactions between two crystalline phases in interpreting DMA experiments, samples with 1 minute annealing times were chosen for active damping in this study in which no traces of minor peak were found. 

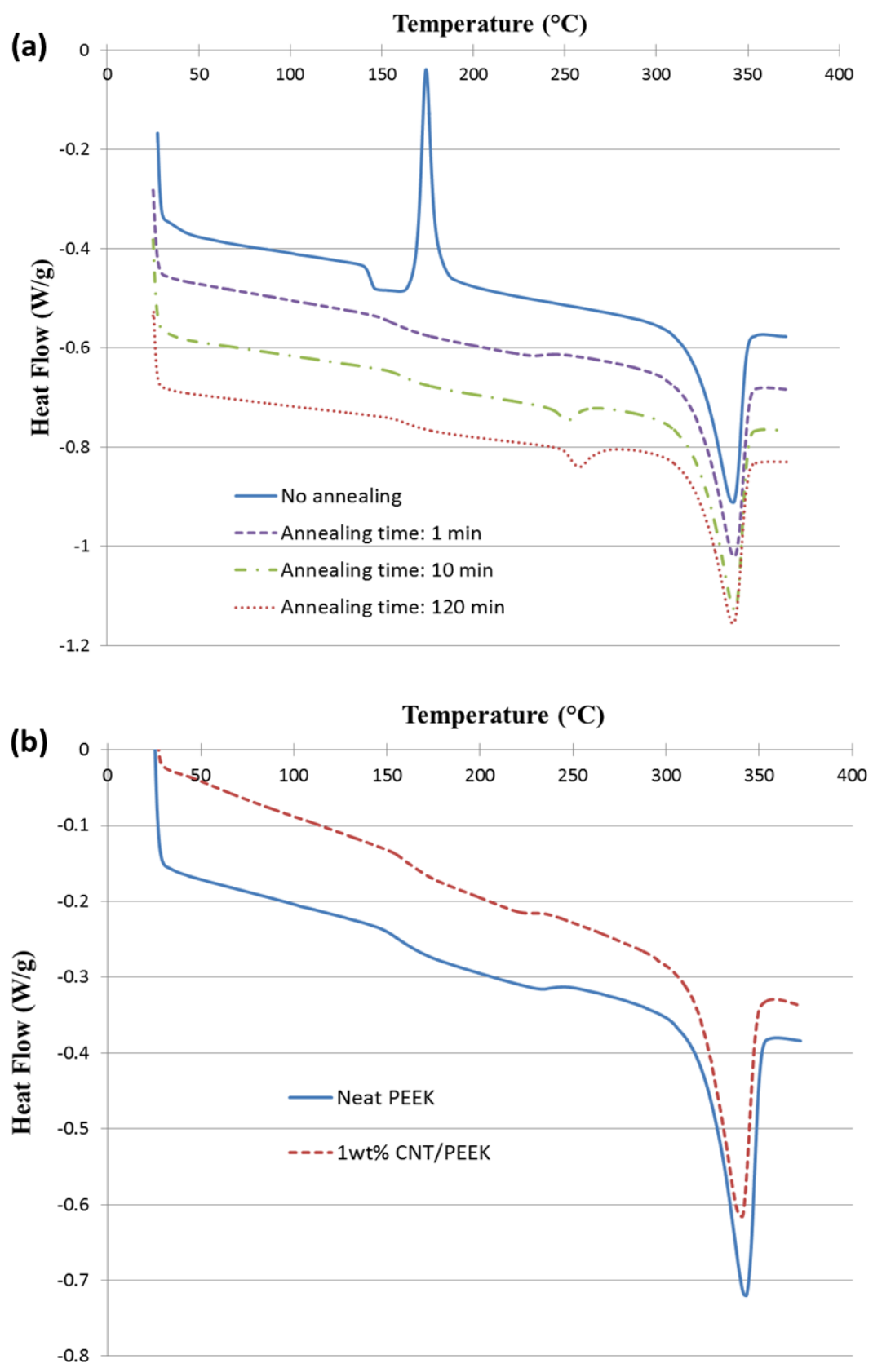

Figure 3. DSC results for (a) neat PEEK samples annealed at $240^{\circ} \mathrm{C}$ for different annealing times. (b) neat PEEK and $1 \mathrm{wt} \%$ CNT/PEEK samples with annealing temperature of $240^{\circ} \mathrm{C}$ for a duration of 1 minute. (The data was offset for clarity). 
Table 1. Degree of crystallinity of PEEK and $1 \mathrm{wt} \%$ CNT/PEEK composite annealed at $240^{\circ} \mathrm{C}$.

\begin{tabular}{|lcccccc|}
\hline Material & $\begin{array}{c}\text { Annealing } \\
\text { conditions }\end{array}$ & $\begin{array}{c}\text { \% crystallinity } \\
\text { (first phase) }\end{array}$ & $\begin{array}{c}\text { \% crystallinity } \\
\text { (second phase) }\end{array}$ & $T_{g}\left({ }^{\circ} \mathbf{C}\right)$ & $\begin{array}{c}\text { Max } \\
\text { tan } \boldsymbol{\delta}\end{array}$ & $\begin{array}{c}\text { E' at RT } \\
(\mathbf{M P a})\end{array}$ \\
\hline PEEK & No annealing & 14.62 & 0 & 145.8 & 1.596 & 1890.9 \\
\hline & $240^{\circ} \mathrm{C}$ for $1 \mathrm{~min}$ & 31.88 & 0 & 159.3 & 0.199 & 2277.8 \\
\hline $\begin{array}{c}240^{\circ} \mathrm{C} \text { for } 10 \\
\text { min }\end{array}$ & 31.88 & 1.56 & 159.6 & 0.177 & 2278.5 \\
\hline $\begin{array}{c}240^{\circ} \mathrm{C} \text { for } 120 \\
\text { min }\end{array}$ & 30.81 & 2.48 & 160.5 & 0.165 & 2538.0 \\
\hline $\begin{array}{l}\mathbf{1 w t} \% \mathbf{C N T} \\
\text { / PEEK }\end{array}$ & \begin{tabular}{c}
$240^{\circ} \mathrm{C}$ for $1 \mathrm{~min}$ \\
\hline
\end{tabular} & 25.50 & 0 & 152.6 & 0.311 & 2049.1 \\
\hline
\end{tabular}

\subsection{Crystallization of PEEK in PEEK/CNT Composites}

The effect of CNTs on the formation of the crystalline phase of PEEK in PEEK/CNT composites was studied via DSC, Figure 3b. As observed in the figure, the addition of CNTs lowers the intensity of the melting peak distinguishably. In other words, crystallization is considerably suppressed with the presence of CNTs. In fact, the degree of crystallization decreased from $31.88 \%$ (neat PEEK) to $25.50 \%$ (1wt\% CNT/PEEK composite) with the addition of $1 \mathrm{wt} \%$ CNTs. The loss in crystallization as a result of the addition of CNTs can be attributed to the spatial limitations imposed on growing crystals due to CNT confinement and disruption of the continuity of polymer matrix during the crystallization process [15, 28]. The reduction in crystallinity was also accompanied with a decrease in $T_{g}$ from $160.5^{\circ} \mathrm{C}$ to $152.6^{\circ} \mathrm{C}$. Interestingly, the $T_{g}$ of CNT/PEEK composites, which are $25.5 \%$ crystalline, falls between the values of $T_{g}$ of the neat PEEK samples with crystallizations of $18.52 \%$ and $31.58 \%$. Hence, the correlation between $\mathrm{Tg}$ and degree of crystallinity observed and presented earlier for neat PEEK can also be extended to CNT/PEEK composites. In other words, the lower crystallinity will pose less limitations on the segmental chain mobility of the transitional chains in between crystalline domains, thus requiring less thermal energy to undergo glass transition.

\subsection{Dependence of Viscoelastic Response of PEEK on Temperature}

The dynamic mechanical properties of neat PEEK and CNT/PEEK composite samples were studied as a function of temperature. By increasing the temperature of the unannealed samples from room temperature, they show a significant drop in storage modulus approaching nearly zero near $T_{g}$. Upon further heating beyond the $T_{g}$, however, the drop in storage modulus is followed by an increase in loss modulus, and the storage modulus of the unannealed sample approaches the value of the annealed samples with considerably higher initial degrees of crystallization. The initial drop of modulus to nearly zero in unannealed samples is expected, since they are highly amorphous and the mechanical behavior of the amorphous phase will dominate. Moreover, the gain in storage modulus above $T_{g}$ is a result of the 
growth of the crystalline phase during the temperature rise in DMA experiments, which is also evidenced in DSC experiments (Figure 3).

In addition, the $\tan \delta$ shows a large peak in unannealed sample (magnitude of 1.596). The magnitude of the peak is significantly reduced in the annealed samples as the degree of crystallization is increased. This trend is expected since the growth of the crystalline domains lowers the mobility of transitional chains in the amorphous phase, as discussed earlier. Thus, crystallization lowers the number of relaxation modes required to dissipate mechanical energy. While the magnitude of tan $\delta$ in annealed samples does not significantly decrease with annealing time, the $T_{g}$ corresponding to maximum $\tan \delta$ shifts to higher temperatures, supporting the notion of restricted movement of the polymer chains by the presence and growth of the crystalline phase. In addition, longer annealing times lead to higher storage moduli, especially near room temperature, by increasing the initial degree of crystallinity, Figure 4.
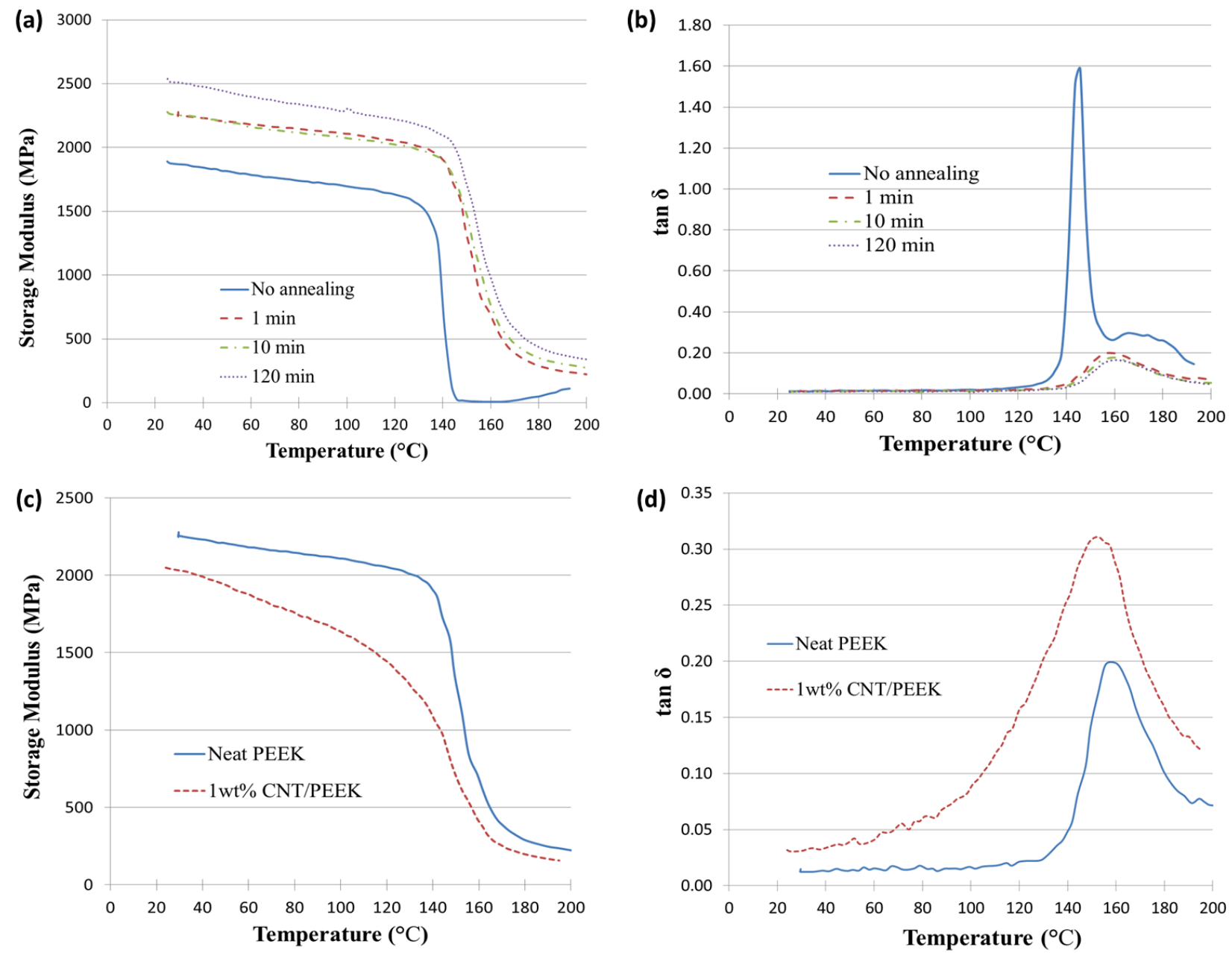

Figure 4. (a) Storage modulus and (b) tan $\delta$ results for neat PEEK samples annealed at $240^{\circ} \mathrm{C}$ for various annealing times. Comparison of (c) storage modulus and (d) $\tan \delta$ between neat PEEK and a $1 \mathrm{wt} \%$ CNT/PEEK composite. 


\subsection{Viscoelastic Response of PEEK/CNT Composites vs. Temperature}

The PEEK/CNT composites were annealed for 1 minute at $240^{\circ} \mathrm{C}$. Compared to the neat PEEK samples with similar duration of annealing, PEEK/CNT had a lower storage modulus (2277.8 MPa compared to 2049.1 MPa, Figure 4c). The lower storage modulus of PEEK/CNT samples is partly rooted in the lower degree of crystallinity of the matrix with the addition of CNTs. Moreover, CNT agglomeration which was observed in SEM images of the fracture surface in our samples can also lower the matrix-CNT interactions leading to poor reinforcing effect and a loss in modulus. The adverse effect of CNT agglomeration on modulus in the present study can further be verified by considering prior studies in which enhancements in modulus of PEEK/CNT composites with CNT uniform dispersion was achieved, where the CNT uniform distribution was obtained by solvent-assisted dispersion or CNT surface functionalization $[15,29]$.

On the other hand, the tan $\delta$ of the CNT composite, Figure 4d, is higher and shows a much broader curve as compared to the neat PEEK sample. The broadening of tan $\delta$ peak can be explained by considering various degrees of chain confinement and mobility due to the presence of CNTs. The spacing between CNTs and polymer chains can vary from angstroms (molecular contact) to tens of nanometers. This distribution of CNT-chain spacing leads to various degrees of confinement on chains. As such, amorphous polymer chains will demonstrate relaxation mechanisms with a wide range of relaxation times and thus glass transition temperatures, leading to a broadened overall $T_{g}$ of the composite [15, 30-33].

\subsection{Active Damping in a CNT/PEEK Composite via Joule Heating}

The ability to adjust and control damping in a $1 \mathrm{wt} \% \mathrm{CNT} / \mathrm{PEEK}$ composite via applied electric power is demonstrated in Figure 5. Prior to the experiment, samples were annealed for $1 \mathrm{~min}$ at $240^{\circ} \mathrm{C}$ to enhance crystallization without developing the minor crystalline phase. As shown in Figure 5a, the damping $(\tan \delta$ ) of the composite increases with increasing input power. Polymer chain relaxation due to increasing temperature caused by Joule heating is the expected damping mechanism. The sharp rise in damping and drop in storage modulus suggests that a large volume of the material is approaching the $T_{g}$ of the matrix, as observed in the thermal images of the powered sample. For instance, as shown in Figure $5 \mathrm{c}$, at an applied power of $0.017 \mathrm{~W} / \mathrm{mm}^{3}$, a large part of the sample is observed to be within $5-10^{\circ} \mathrm{C}$ of $T_{g}$. The onset of detectable enhancement in $\tan \delta$ (power per unit volume of $\sim 0.012 \mathrm{~W} / \mathrm{mm}^{3}$ ) correlates with a drop in storage modulus, Figure $5 \mathrm{~b}$. In addition, the inset temperature distribution images in Figure $5 \mathrm{~b}$ show that the average temperature in the sample at the onset of $\tan \delta$ enhancement is about $100^{\circ} \mathrm{C}$. According to our DMA studies, Figure $4 \mathrm{~d}$, the broadening of the tan $\delta$ curve initiates at around $100^{\circ} \mathrm{C}$ (the change in slope of the tan $\delta$ curve occurs around this temperature). Hence, the onset of enhancement of $\tan \delta$ with input power during Joule heating experiment can be attributed to thermal activation of viscous mechanisms and chain relaxation. During the course of the experiment, the $\tan \delta$ increases by a factor of 4 , with an average sample temperature reaching $115.4^{\circ} \mathrm{C}$. The $400 \%$ increase in damping is achieved at a much lower relative loss $(\sim 42 \%)$ in storage modulus, demonstrating a favorable trade-off between damping augmentation and structural stiffness. In other words, while the crystalline phase maintains considerable stiffness (manifested in the residual storage modulus near $T_{g}$ ), the amorphous phase contributes to energy dissipation through segmental movements of polymer chains. The effectiveness of the active damping was evaluated by calculating the variation of the figure of merit for 
damping materials, which was defined by Lakes [19] as the product of the absolute value of the complex modulus and the $\tan \delta$. As shown in Figure 5(d), the figure of merit of the composite monotonically increases with input power, such that at the maximum power, it has increased by $\sim 150 \%$ compared to its value at room temperature.

However, temperature non-uniformity, in both micro and macroscale (discussed in section 3), will lower the effectiveness of active damping. The adverse effect of non-uniform temperature distribution on active damping can be highlighted by considering a PEEK/CNT sample in which the average temperature is raised near $T_{g}$ via Joule heating. The wider the distribution of temperature, the larger the overheated and underheated (respectively hotter and colder than $T_{g}$ ) portions of the sample. However, in the overheated regions, the $\tan \delta$ is lower than the maximum (the tail of the $\tan \delta$ vs. T curve at temperatures higher $T_{g}$ ). Hence, wider temperature distributions will lower the effective tan $\delta$ of Joule heated sample. The presence of a crystalline phase is specifically critical in overheated regions, since it limits the loss in storage modulus.
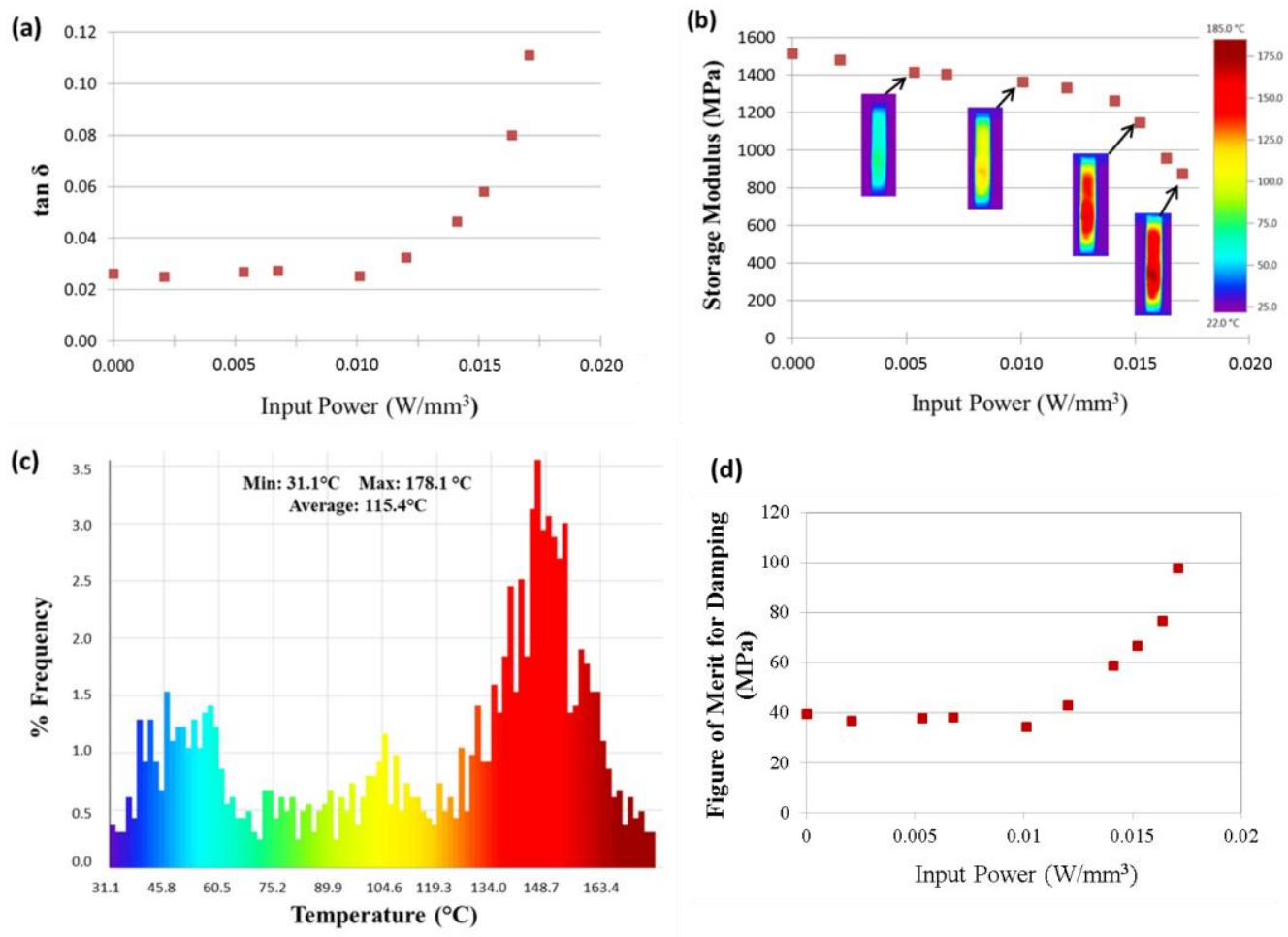

Figure 5. (a) $\tan \delta$ and (b) storage modulus of a $1 \mathrm{wt} \%$ CNT/PEEK composite as a function of applied power. Inset images in plot (b) show the temperature distribution on the sample surface at different power inputs. (c) Histogram showing the temperature distribution in a $1 \mathrm{wt} \%$ CNT/PEEK composite for an applied power of $0.017 \mathrm{~W} / \mathrm{mm}^{3}$. (d) Figure of merit for damping of the PEEK/CNT nanocomposite as a function of input power per unit volume

Another adverse effect of the non-uniform temperature distribution in the Joule heated samples is that overheated portions of the sample may thermally degrade. In order to test whether increased local heating has resulted in thermal degradation of the polymer, the viscoelastic properties of the composite were measured at room temperature before and after joule heating. The results presented in Figure 6 show that 
both the storage modulus and $\tan \delta$ fully recover to their value before joule heating. This result indicates that the composite sample did not experience any noticeable permanent damage or thermal degradation as a result of resistive heating of the nanoparticles. In addition, this result shows that the active damping mechanism in the composite is reversible.
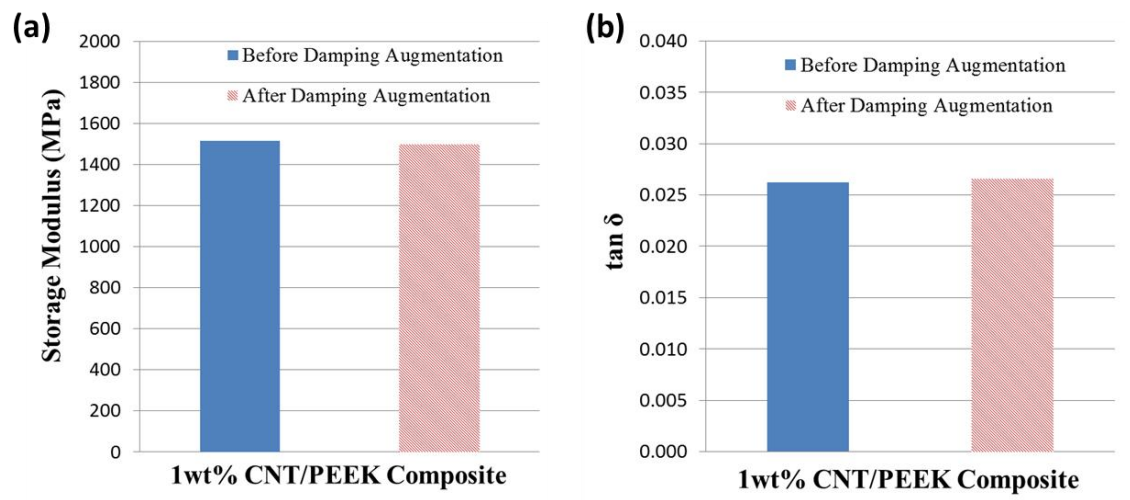

Figure 6. (a) storage modulus and (b) $\tan \delta$ of a $1 \mathrm{wt} \%$ CNT/PEEK composite before and after active damping tests where joule heating was performed to near the $T_{g}$ of the composite.

\subsection{Continuum modeling of non-uniform Temperature distribution in active damping}

The temperature distribution in the sample at the highest applied power obtained from the thermographs of the heated sample, Figure 5c, shows that a large portion of the sample is considerably below or above $T_{g}$. The lower temperature regions along the peripheries of the sample arise from the temperature gradient imposed by the tensile grips, while the high temperature spots may arise from a nonuniform distribution of CNTs. To estimate the effect of macroscale non-uniform temperature distribution on damping, the heated sample was approximated as several viscoelastic segments in series and parallel with different viscoelastic properties depending on the temperature of the segment to be input from DMA experiments (method explained in section 3.1). The variations in viscoelastic properties were caused by a non-uniform temperature distribution, measured from thermal images of the sample, such as the ones shown in Figure 5b. Each image was divided into seven sections by visually identifying regions with a narrow temperature distribution, as shown in Figure $7 \mathrm{a}$ and $\mathrm{b}$. A combination of Voigt and Reuss structures were used to calculate the effective $\tan \delta$ of the composite. With the loading direction being parallel to the longest sample dimension, sections 1, 2, and 3 are in parallel with each other and in series with sections $4,5,6$, and 7 . 

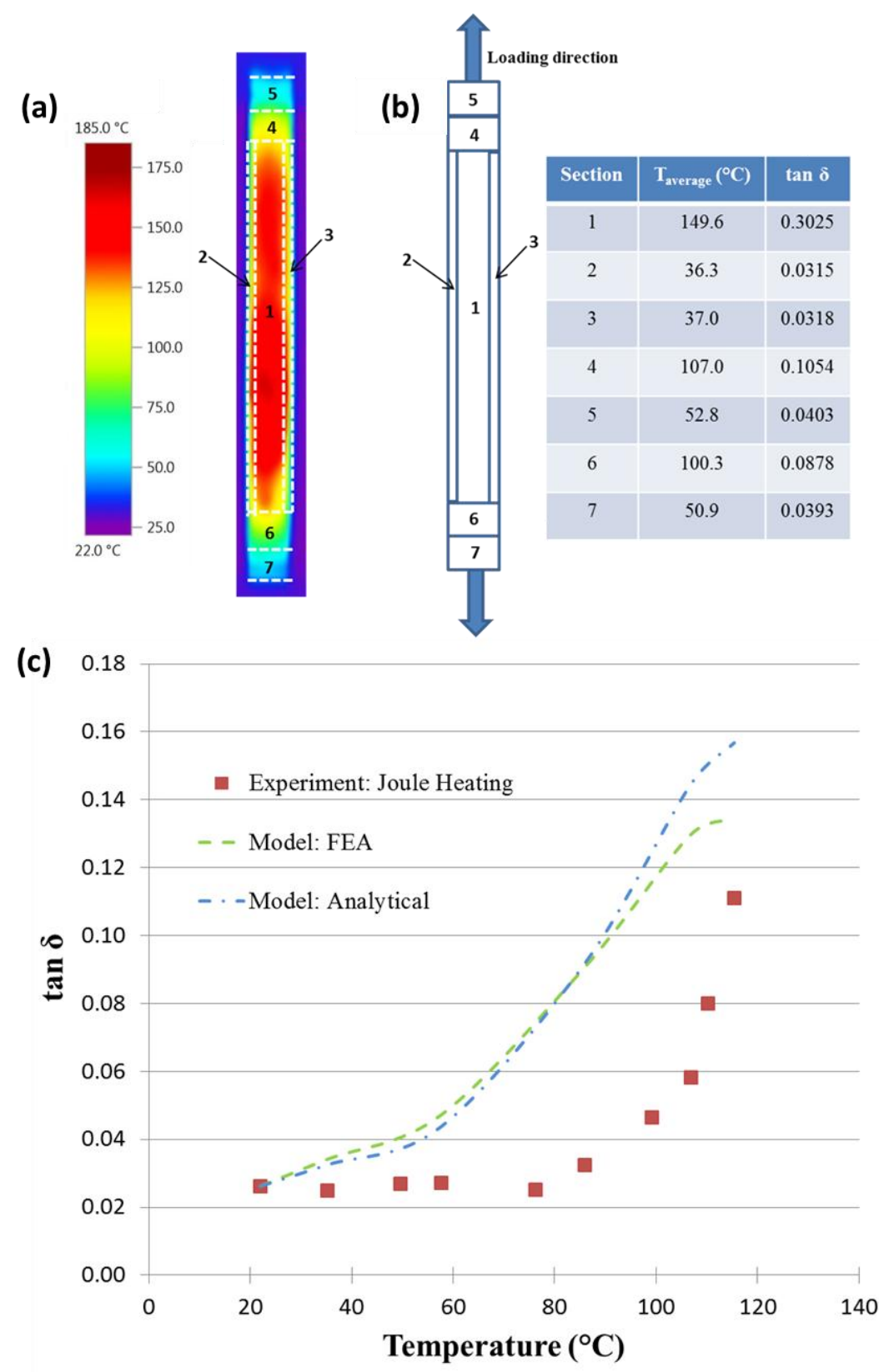

Figure 7. (a) Thermal image of composite sample showing the sample divided into seven segments corresponding to a different temperature (b) Corresponding segments with sections 1 , 2 , and 3 being in parallel to each other and in series with sections $4,5,6$, and 7 . The average temperature, $\mathrm{T}_{\text {average, }}$ and corresponding $\tan \delta$ is shown for each section. (c) Comparison of $\tan \delta$ vs Temperature between modeling and experimental results. (The temperature corresponds to the average temperature of the sample.)

A comparison between the effective $\tan \delta$ of the analytical model and experiment for the $1 \mathrm{wt} \%$ CNT/PEEK composite is shown in Figure 7c. The prediction of damping from the FEA model (the method discussed in section 3.2) is also plotted in the figure. The tan $\delta$ for both models is plotted as a function of the average temperature of the sample. 
The FEA and analytic model agree very well, indicating the accuracy of Voigt and Reuss models in predicting the viscoelastic behavior of the sample with non-uniform temperature. At average temperatures above $100{ }^{\circ} \mathrm{C}$, the finite element analysis deviates slightly from the analytical model. The difference between the two could arise from the point-wise temperature distribution in the FEA analysis, as compared to the approximation of a single temperature for each sections in the analytical model. Since the temperature sensitivity is greater at higher temperatures, the FEA analysis is expected to yield a more reliable result. Moreover, both models qualitatively follow the trend observed in the active damping experiment (Joule heating). However, the models do not quantitatively capture the behavior observed in the joule heating experiments.

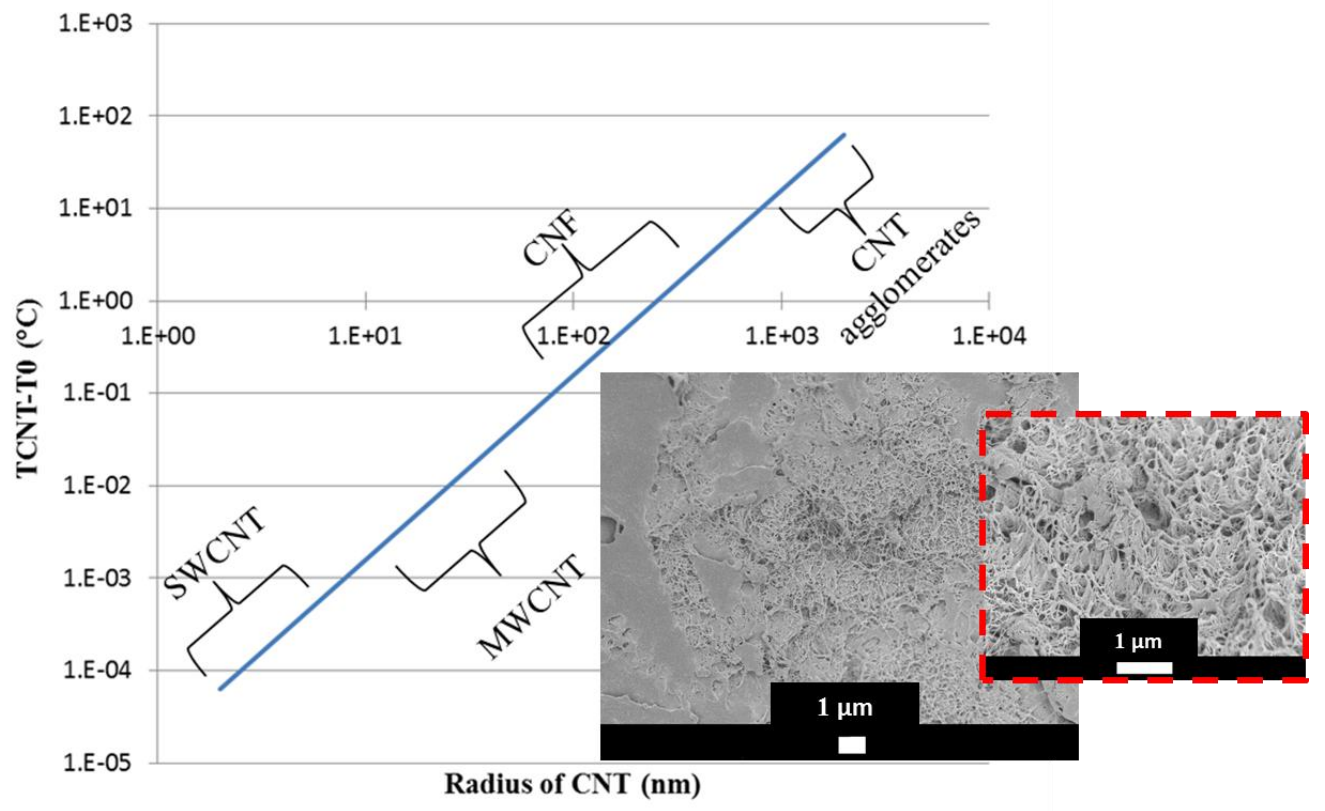

Figure 8. Effect of effective filler radius on the temperature difference between the CNT boundary and the outer boundary of building block of a $1 \mathrm{wt} \% \mathrm{CNT} / \mathrm{PEEK}$ composite. Inset shows SEM image of CNT agglomeration. A zoomed imaged of an agglomerate is shown, outlined by a red dashed line.

To investigate the origin of the discrepancy between the model and experiment, we estimated temperature gradients at the microscale. However, the microscale temperature gradients were not captured by the thermal camera, and it was estimated following the approach outlined in section 3.3. The effect of CNT radius on the temperature difference in the matrix, the second term of Eq. (9), is shown in Figure 8. As observed in the plot, the temperature change across the matrix phase in the radial direction increases linearly with an increase in filler radius. Small diameter fillers, such as single-wall carbon nanotubes (SWCNT) show a rather uniform temperature in the matrix. However, CNT agglomeration, which increase the effective CNT diameter, can lead to large temperature gradients and temperature nonuniformities in the matrix at the microscale [34]. It is also worth noting the effect of CNT agglomeration on temperature non-uniformity. To this end, we considered an effective diameter of CNTs in the high agglomeration regions to be about the diameter of the CNT agglomerates. From SEM images, this 
diameter can be as high as a few microns (inset in Figure 8). For a CNT particle with such high effective diameter, the model predicts a temperature gradient of $\sim 10-50^{\circ} \mathrm{C}$ (Figure 8 ). This gradient of temperature is comparable to the full width at half maximum (FWHM) of the $\tan \delta$ vs. Temperature curve of the composite (Figure 4d). Such high values of local temperature changes in the matrix suggests that the mean temperature of the region may reach $T_{g}$ only if a large portion of the sample is heated to a temperature above $T_{g}$. Thus, a loss in both storage modulus and $\tan \delta$ is inevitable. It is, however, to be pointed out that such microscale temperature gradients occur at length scales below the spatial resolution of the thermal camera and thus cannot be detected experimentally.

Based on the modeling works and the comparison between model predictions and experimental results, in a sample which is experiencing typical cooling mechanisms such as air convection, the most critical factor determining the efficiency of active damping is the dispersion and diameter of the fillers. As shown in Figure 8, incorporating other fillers such as MWCNT or CNF will lead to a higher temperature gradient in the matrix and thus result in more pronounced temperature non-uniformity in the composite. However, if the filler dispersion is uniform, this temperature distribution could remain sufficiently close to $T_{g}$. As shown in Figure 8, to keep the local temperature uniformity within a threshold of $\sim 10^{\circ} \mathrm{C}$ (which is about the FMHW of $T_{g}$ vs. Temperature curves of a typical polymer) the effective particle size should be below $\sim 1 \mu \mathrm{m}$ in radius. However, one drawback is that as filler diameter increases so does the electrical percolation threshold, requiring higher volume fractions to achieve a conductive network. This would also be the case for spherical particles. Therefore, ideally, large aspect ratio fillers of small diameter (tens of nanometers) will provide the most uniformity of temperature at a low volume fraction. This can be achieved by incorporating well dispersed nanoparticles such as CNTs. Dispersion is of significant importance because particle agglomeration will increase the intensity of the heat generated locally, elevating the temperature gradient around the agglomerate above the threshold.

\section{Conclusion}

The concept of active damping in semi-crystalline thermoplastics (PEEK) which are reinforced with a percolated network of CNTs was studied, where the damping of the composite was augmented considerably, controllably and reversibly via Joule heating. The tan $\delta$ in the composite showed an enhancement of up to $400 \%$, which was accompanied with an undesired, but much smaller, drop in storage modulus (by 42\%), corresponding to over $150 \%$ improvement in the figure of merit for damping materials. The semi-crystalline nature of PEEK allows for damping enhancement while retaining a considerable portion of the storage modulus due to the presence of the crystalline phase. No thermal degradation of the polymer was observed and the damping mechanism was found to be reversible, with

both the storage modulus and $\tan \delta$ fully recovering their room temperature values upon cooling of the composite.

The total enhancement in energy dissipation was limited by the fact that the temperature distribution in the sample was not uniform. The temperature non-uniformity was measured experimentally in the macroscale and was estimated via continuum heat transfer models in the microscale. In the microscale, it was found that the spatial temperature variations in the polymer matrix scales with the effective diameter of the filler. Even though the composite system studied here is made up of CNT with small diameters $(<10 \mathrm{~nm})$ the presence of agglomeration can lead to non-uniformity of temperature in the matrix of as much as $\sim 10-50^{\circ} \mathrm{C}$ (in comparison to the $\mathrm{FWHM}$ of the $T_{g}$ peak in PEEK of $\sim 10-20^{\circ} \mathrm{C}$ ). With such high 
temperature variations, the mean temperature of the region surrounding CNT agglomerates may not reach $T_{g}$ unless a large portion of the sample is heated above or below $T_{g}$, both of which will lower the effective tan $\delta$ of the sample. This work outlines the significance of active damping control in nanocomposites using a percolated network of CNTs, pointing to the importance of uniform dispersion of CNTs to achieve the highest enhancement in effective $\tan \delta$ of the composite.

\section{Acknowledgements}

The authors would like to thank Dr. Hung-Jue Sue at Texas A\&M University for providing the neat PEEK material. In addition, M.N. and D.C.L. would like to acknowledge the support of DOD-Army Research Laboratory under the award No. W911NF-14-2-0080. The use of the TAMU Materials Characterization Facility for SEM imaging is acknowledged.

\section{References}

1. Wetton, R., R. Marsh, and J. Van-de-Velde, Theory and application of dynamic mechanical thermal analysis. Thermochimica Acta, 1991. 175(1): p. 1-11.

2. Glaz, B., et al., Interfacial Strain Energy Dissipation in Hybrid Nanocomposite Beams Under Axial Strain Fields. AIAA Journal, 2015. 53(6): p. 1544-1554.

3. Aiken, E.W., R.A. Ormiston, and L.A. Young. Future directions in rotorcraft technology at ames research center. in 56th American Helicopter Society Annual Forum. 2000. Virginia Beach, VA.

4. Sun, L.Y., et al., Energy absorption capability of nanocomposites: A review. Composites Science and Technology, 2009. 69(14): p. 2392-2409.

5. Sierakowski, R.L., I.Y. Telitchev, and O.I. Zhupanska, On the impact response of electrified carbon fiber polymer matrix composites: effects of electric current intensity and duration. Composites Science and Technology, 2008. 68(3): p. 639-649.

6. Barakati, A. and O. Zhupanska, Thermal and mechanical response of a carbon fiber reinforced composite to a transverse impact and in-plane pulsed electromagnetic loads. Journal of Engineering Materials and Technology, 2012. 134(3): p. 031004.

7. Cimellaro, G.P., Simultaneous stiffness-damping optimization of structures with respect to acceleration, displacement and base shear. Engineering Structures, 2007. 29(11): p. 2853-2870.

8. Finegan, I.C. and R.F. Gibson, Recent research on enhancement of damping in polymer composites. Composite Structures, 1999. 44(2): p. 89-98.

9. Gardea, F., et al., Energy Dissipation Due to Interfacial Slip in Nanocomposites Reinforced with Aligned Carbon Nanotubes. ACS Applied Materials \& Interfaces, 2015. 7(18): p. 9725-9735.

10. Schadler, L., L. Brinson, and W. Sawyer, Polymer nanocomposites: a small part of the story. JOM, 2007. 59(3): p. 53-60. 
11. Gardea, F., M. Naraghi, and D.C. Lagoudas, Effect of thermal interface on heat flow in carbon nanofiber composites. ACS Applied Materials \& Interfaces, 2013. 6(2): p. 10611072 .

12. Gardea, F. and D.C. Lagoudas, Characterization of electrical and thermal properties of carbon nanotubelepoxy composites. Composites Part B: Engineering, 2014. 56: p. 611620.

13. Smith, K.E., J.R. Maly, and C.D. Johnson. Smart tuned mass dampers. in Active Materials and Adaptive Structures: Proceedings of the ADPA/AIAA/ASME/SPIE Conference. 1991.

14. Searle, O.B. and R.H. Pfeiffer, Victrex ${ }^{\circledR}$ poly(ethersulfone) (PES) and Victrex ${ }^{\circledR}$ poly(etheretherketone) (PEEK). Polymer Engineering \& Science, 1985. 25(8): p. 474476.

15. Díez-Pascual, A.M., et al., Development and characterization of PEEK/carbon nanotube composites. Carbon, 2009. 47(13): p. 3079-3090.

16. Díez-Pascual, A.M., et al., Novel nanocomposites reinforced with hydroxylated poly (ether ether ketone)-grafted carbon nanotubes. Journal of Materials Chemistry, 2010. 20(38): p. 8247-8256.

17. Denault, J. and M. Dumouchel, Consolidation Process of PEEK/Carbon Composite for Aerospace Applications. Advanced Performance Materials. 5(1): p. 83-96.

18. Kurtz, S.M. and J.N. Devine, PEEK biomaterials in trauma, orthopedic, and spinal implants. Biomaterials, 2007. 28(32): p. 4845-4869.

19. Lakes, R.S., High damping composite materials: Effect of structural hierarchy. Journal of Composite Materials, 2002. 36(3): p. 287-297.

20. Onoda, J., et al., Vibration Suppression by Variable-Stiffness Members. Aiaa Journal, 1991. 29(6): p. 977-983.

21. Rae, P., E. Brown, and E. Orler, The mechanical properties of poly (ether-etherketone)(PEEK) with emphasis on the large compressive strain response. Polymer, 2007. 48(2): p. 598-615.

22. Kong, Y. and J. Hay, The measurement of the crystallinity of polymers by DSC. Polymer, 2002. 43(14): p. 3873-3878.

23. Bassett, D., R. Olley, and I. Al Raheil, On crystallization phenomena in PEEK. Polymer, 1988. 29(10): p. 1745-1754.

24. Chen, C. and R. Lakes, Analysis of high-loss viscoelastic composites. Journal of Materials Science, 1993. 28(16): p. 4299-4304.

25. Hashin, Z., Complex moduli of viscoelastic composites-I. General theory and application to particulate composites. International Journal of Solids and Structures, 1970. 6(5): p. 539-552. 
26. Verma, R., H. Marand, and B. Hsiao, Morphological changes during secondary crystallization and subsequent melting in poly (ether ether ketone) as studied by real time small angle X-ray scattering. Macromolecules, 1996. 29(24): p. 7767-7775.

27. Lattimer, M., et al., On the origin of the multiple endotherms in PEEK. Polymer, 1992. 33(18): p. 3971-3973.

28. Kodjie, S.L., et al., Morphology and crystallization behavior of HDPE/CNT nanocomposite. Journal of Macromolecular Science, Part B: Physics, 2006. 45(2): p. 231245.

29. Sandler, J., et al., Carbon-nanofibre-reinforced poly (ether ether ketone) composites. Composites Part A: Applied Science and Manufacturing, 2002. 33(8): p. 1033-1039.

30. Miaudet, P., et al., Shape and temperature memory of nanocomposites with broadened glass transition. Science, 2007. 318(5854): p. 1294-1296.

31. Díez-Pascual, A.M., et al., High-performance nanocomposites based on polyetherketones. Progress in Materials Science, 2012. 57(7): p. 1106-1190.

32. Sung, Y., et al., Dynamic mechanical and morphological properties of polycarbonate/multi-walled carbon nanotube composites. Polymer, 2005. 46(15): p. 5656-5661.

33. Tsagaropoulos, G. and A. Eisenberg, Dynamic mechanical study of the factors affecting the two glass transition behavior of filled polymers. Similarities and differences with random ionomers. Macromolecules, 1995. 28(18): p. 6067-6077.

34. Martin, A.C., et al., Amorphous-to-crystalline transition of Polyetheretherketone-carbon nanotube composites via resistive heating. Composites Science and Technology, 2013. 89: p. 110-119. 